\title{
Formant transitions in fricative identification: The role of native fricative inventory
}

\author{
Anita Wagner, ${ }^{a)}$ Mirjam Ernestus, and Anne Cutler \\ Max Planck Institute for Psycholinguistics, Nijmegen, 6500 AH, The Netherlands
}

(Received 1 August 2005; revised 28 June 2006; accepted 14 July 2006)

\begin{abstract}
The distribution of energy across the noise spectrum provides the primary cues for the identification of a fricative. Formant transitions have been reported to play a role in identification of some fricatives, but the combined results so far are conflicting. We report five experiments testing the hypothesis that listeners differ in their use of formant transitions as a function of the presence of spectrally similar fricatives in their native language. Dutch, English, German, Polish, and Spanish native listeners performed phoneme monitoring experiments with pseudowords containing either coherent or misleading formant transitions for the fricatives /s/ and /f/. Listeners of German and Dutch, both languages without spectrally similar fricatives, were not affected by the misleading formant transitions. Listeners of the remaining languages were misled by incorrect formant transitions. In an untimed labeling experiment both Dutch and Spanish listeners provided goodness ratings that revealed sensitivity to the acoustic manipulation. We conclude that all listeners may be sensitive to mismatching information at a low auditory level, but that they do not necessarily take full advantage of all available systematic acoustic variation when identifying phonemes. Formant transitions may be most useful for listeners of languages with spectrally similar fricatives. (C) 2006 Acoustical Society of America. [DOI: 10.1121/1.2335422]
\end{abstract}

PACS number(s): 43.71.Es, 43.71.Hw [ARB]

Pages: 2267-2277

\section{INTRODUCTION}

Do formant transitions contribute to listeners' identification of fricatives? These dynamic cues are crucial for the identification of stops, but despite decades of research (Harris, 1958; Heinz and Stevens, 1961; LaRiviere, Winitz, and Herriman, 1975; Jongman, 1989; Jongman, Wayland, and Wong, 2000), no clear answer has emerged for fricatives. Salient static cues are present in the fricative spectrum, and may suffice for phoneme identification. We report a study which contributes to this discussion by testing the hypothesis that the contribution of formant transitions is language specific and depends on the presence of spectrally similar fricatives in the listener's native phoneme inventory.

Fricatives are produced with a narrow constriction in the oral cavity. The turbulence of the airflow passing this constriction generates the characteristic sound of frication. The exact location of the narrow passage and the size and form of the cavity in front of the constriction define the acoustic characteristics of the fricative (Stevens, 1998). These energy peaks and minima in a fricative's spectrum serve listeners as primary cues for fricative identification (Stevens, 1998). The salience of those spectral poles, however, differs among fricatives, and previous research (e.g., Harris, 1958) suggests that listeners need additional cues to identify some but not all fricatives. Whereas sibilants have very pronounced spectral peaks and are identified primarily on the basis of these poles, dental and labiodental fricatives have a more diffuse energy spectrum and may require additional cues for accurate identification. Two contextual sources of such cues have been

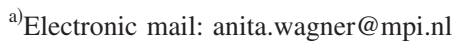

found (Whalen, 1981): formant transitions, which may be perceptually integrated with cues from the fricative spectrum; and the quality of the surrounding vowels, including the resulting slight modifications of the fricative spectrum itself.

It is unclear, however, whether formant transitions indeed contribute to the identification of fricatives, since the results from previous research are conflicting. Harris (1958) studied the identification of English fricatives in different vocalic contexts. In a fricative categorisation experiment, she presented American students with natural tokens of consonant (C) vowel (V)-syllables containing the fricatives /f v $\theta$ ð s z $\int 3 /$ combined with the vowels /a I u e/. These syllables were spliced such that every fricative was combined with every vowel as produced in the context of each of the fricatives. Thus, the formant transitions in some tokens contained misleading information with respect to the identity of the fricative. Participants accurately categorized $/ \mathrm{s} /$ and $/ \mathrm{S} /$ in the combination of just the frication part from the sibilant with each of the vowels, independently of the fricative context from which these vowels were extracted. In contrast, stimuli with frication from $/ \mathrm{f} /$ or $/ \theta /$ were often confused with each other. In fact, the /f/ tended to be categorized as /f/ only when combined with a vowel originally produced after $/ \mathrm{f} /$, but as $/ \theta /$ when followed by any other vowel. Apparently, the English listeners recognized the sibilants/s/ and / / by their frication part alone, while the dental fricatives $/ \mathrm{f} /$ and $/ \theta /$ were accurately categorized only when followed by correct formant transitions.

Similar results were obtained by Heinz and Stevens (1961) with synthesized English voiceless fricatives. American listeners identified /s $\int \mathrm{f} \theta /$ in isolation, and achieved satisfactory identification rates for $/ \mathrm{s} \int /$, but they could not 
distinguish between /f/ and / $\theta /$. The identification scores improved when the fricatives were combined with the synthetic vowel /a/, including approximated transition movements; especially the distinction between /f/ and / $\theta /$ was more reliably perceived.

More recent studies, however, failed to replicate these results. Jongman (1989) asked English listeners to identify fricatives by listening either to portions of the frication alone, or to the whole frication, or to complete syllables (all eight English fricatives except /h/; produced by an American speaker with the vowels /a i u/). A portion of the frication longer than $40 \mathrm{~ms}$ appeared to be sufficient for listeners to identify all fricatives accurately, including the oft-confused fricatives $/ \mathrm{f} /$ and $|\theta|$. No improvement of fricative identification resulted from inclusion of the vowel. Jongman et al. (1998) further supported this conclusion in a production study. They analyzed the variances of locus equations (Fruchter and Sussman, 1997) of English fricatives followed by the vowels /i e æ a o $\mathrm{u} /$ as produced by 20 speakers. On this parameter /f $\mathrm{v} /$ differed significantly from /s z $\int 3 \theta$ d/, but the three places of articulation represented in the latter set did not differ. Jongman et al. (1998) concluded that locus equations cannot sufficiently cue fricative place of articulation.

LaRiviere, Winitz, and Herriman (1975), too, queried the role of formant transitions in fricative identification. They compared identification of syllables made up of /f $\theta \mathrm{s} \mathrm{J} /$ and /a i u/, with the identification of the same syllables with deleted formant transitions. Listeners could reliably identify all fricatives in transitionless syllables, and the authors thus concluded that formant transitions do not necessarily contribute to fricative identification. LaRiviere et al. also found that $/ \theta /$ was the most difficult fricative to identify. They explain possible, but not necessary, perceptual benefit from the following vowel as arising from the information that it carries about the speaker's vocal tract, which contributes to the process of speaker normalisation.

Klaassen-Don (1983) also found no evidence that formant transitions contribute to fricative identification. In a gating experiment with Dutch fricatives, she presented naturally produced $\mathrm{CV}$ and $\mathrm{VC}$ strings including the fricatives /f v s z $\int \mathrm{x} /$ and the vowels / $\mathrm{a} \mathrm{i}$ u/. The syllables were produced in isolation or were excerpted from running speech. Formant transitions proved to be valuable cues for liquids and stops, but their contribution in fricative identification was negligible. Klaassen-Don reached the conclusion that "vowel transitions do not contain perceptually relevant information about adjacent fricatives in Dutch" (KlaassenDon, 1983, p. 79).

Finally, in a series of production and perception experiments, Borzone de Manrique and Massone (1981) investigated the identification of Argentinian Spanish fricatives by native listeners. The perceptual power of the most prominent noise frequency bands was tested by bandpass filtering the fricatives $/ \mathrm{s} \mathrm{f} \int \mathrm{x} /$. The identifications showed that $/ \mathrm{s} /$ is the most robust fricative, whereas /f/ requires a wide noise band to be accurately identified. In further experiments, the authors concentrated on the role of the vocalic environment for fricative identification by Argentinian listeners. Their stimuli consisted of frication and vocalic parts spliced out of naturally produced $\mathrm{CV}$ syllables and of transitionless CV syllables, which they constructed by combining natural fricatives and vowels produced in isolation. For Argentinian listeners the frication part alone was sufficient to identify all fricatives, with the exception of the velars $/ x \mathrm{\gamma} /$. The $a b-$ sence of transitions in the vowel biased the listeners to the fricative that is realized with the least transition movements into the following vowel. For instance, the formant transitions following /f/ are shorter before / $\mathrm{u} /$ than before /i/, and the authors observed a higher number of /f/ categorizations for syllables consisting of frication and $/ \mathrm{u} /$ rather than frication and $/ \mathrm{i} /$.

In short, the literature shows that formant transitions proved to be useful cues in some experiments but of little use in others. Importantly, the experiments involved listeners of different native languages. We hypothesize that the solution to the conflicting results is that listeners' attention to formant transitions for fricative identification is language specific and modulated by the presence of perceptually similar fricatives in the native phoneme inventory. Languages differ widely in how many fricatives they include, and how similar these fricatives are. More fricatives in a given perceptual space may reduce the distinctiveness of individual fricatives. To maximize the distinctiveness of fricatives in denser perceptual spaces, listeners may learn to integrate additional cues to attain accurate percepts of these fricatives.

If listeners of different native languages indeed differ in the use they make of transitional cues, we can further ask whether listeners who do exploit transitional information do so for all native fricatives, or only for contrasts which are perceptually similar. Listeners' language experience may tune the perceptual system to select relevant cues efficiently for each fricative: If more salient cues suffice to distinguish a given phoneme contrast, native listeners may make no use of the information in formant transitions. Thus our second hypothesis is that attention to formant transitions can be restricted to those fricatives that are difficult to distinguish spectrally. The fricative pair /f $\theta /$ seems, on the evidence cited above, to be difficult to distinguish for English listeners. For Argentinian listeners, without /f $\theta /$ in their native phoneme inventory, a different pair of fricatives appears to be potentially confusable: $/ \mathrm{x} \mathrm{\gamma} /$. We assume that listeners will learn the most efficient way to identify all native fricatives, and that it might not be beneficial for them to use the cues in formant transitions for fricatives that can be identified accurately on the basis of the fricative spectrum alone.

In the present study, listeners of different languages heard pseudowords containing either coherent or misleading information in the formant transitions surrounding fricatives. In four experiments participants performed phoneme monitoring, a task that has been used to investigate a wide range of psycholinguistic issues (see Connine and Titone, 1996, for a review). In phoneme monitoring, listeners hear spoken input, e.g., lists of words, nonwords, or syllables, and respond as soon as they detect a prespecified target phoneme. Phoneme monitoring is especially promising as a paradigm for testing our hypothesis because it has been shown to be sensitive to formant transitions: Detection of a phoneme is more 
TABLE I. The fricative inventories of the languages studied according to the place of articulation.

\begin{tabular}{|c|c|c|c|c|c|c|c|c|}
\hline & Labiodental & Dental & Alveolar & Postalveolar & Retroflex & Alveolopalatal & Velar & Glottal \\
\hline Dutch & $\mathrm{f} \mathrm{v}$ & & S Z & $\left(\int\right)$ & & & $\mathrm{X}$ & $\mathrm{h}$ \\
\hline German & $\mathrm{f} \mathrm{v}$ & & s z & $\int 3$ & & & $\mathrm{X}$ & $\mathrm{h}$ \\
\hline Spanish & $\mathrm{f}$ & $\theta$ & $\mathrm{s}$ & & & & $\mathrm{X}$ & \\
\hline English & $\mathrm{f} \mathrm{v}$ & $\theta$ ð & s Z & $\int 3$ & & & & $\mathrm{~h}$ \\
\hline Polish $^{1}$ & $\mathrm{f} \mathrm{v}$ & & s z & $\int^{j} 3^{j}$ & $\mathrm{~S} \mathrm{Z}$ & 67 & $\mathrm{x}$ & \\
\hline
\end{tabular}

${ }^{2}$ Polish postalveolar fricatives $/ \int 3 /$ are traditionally described as laminal alveolar (Jassem, 2003), and the alveolopalatal / $\mathrm{z} / \mathrm{are}$ considered as their palatalized counterparts. Hamann (2003) argues that Polish postalveolar fricatives should be considered as retroflex; in addition Zygis and Hamann (2003) claim that the alveolopalatal and the palatalized postalveolar fricatives in Polish should be considered two separate sounds, as they are distinguished by native and non-native listeners. This view is adopted in our description of the Polish fricative repertoire.

difficult when its context is cross spliced and thus bears mismatching coarticulatory information (Martin and Bunnell, 1981; McQueen, Norris, and Cutler, 1999). Moreover, the task is sensitive to cross-language differences in speech processing. Otake et al. (1996) and Weber (2001) showed effects of language-specific phonotactic constraints in phoneme monitoring for nasals and fricatives, respectively. Similarly, with the same task Costa, Cutler, and SebastiánGallés (1998) showed that processing of acoustic variation is affected by native phoneme inventory constitution.

If listeners depend on formant transitions in fricative identification, then mismatching formant transitions should increase errors and slow reaction times in phoneme monitoring. In contrast, listeners whose fricative identification is governed mostly by the primary static cues in the noise spectrum should be less affected by misleading formant transitions, either in reaction speed or error rate.

We tested five languages: German and Dutch, which both have only spectrally distinct fricatives, and (Castilian) Spanish, English, and Polish, which all have pairs of fricatives in which the distribution of noise peaks across the spectrum is very similar, so that the members of the pair are perceptually less distinctive. Spanish and English contrast with Polish with respect to which spectrally similar fricatives appear in the phoneme inventory. Table I sketches the fricative inventories of the five languages.

Experiment I contrasted Spanish with Dutch and German. Spanish, as we saw, has the confusable pair /f $\theta /$. The spectra of the labiodental and dental fricatives are relatively flat; the energy is distributed in each case across frequencies from circa $2-10 \mathrm{kHz}$ with no defined spectral peaks (Jongman, Wayland, and Wang, 2000). We therefore expected Spanish listeners to pay more attention to formant transitions than Dutch or German listeners, whose languages contain no spectrally similar fricatives. The fricatives in the experiment were the labiodental /f/ and the alveolar/s/. Since of these only /f/ is spectrally confusable with another fricative in Spanish, we further expected Spanish listeners to be particularly affected by mismatching formant transitions for /f/.

\section{EXPERIMENT I}

\section{A. Method}

\section{Materials}

Three- and four-syllable pseudowords made up of the phonemes /p b t d k f s a i u e/ (e.g., tikusa and doku- pafi) were recorded by a native speaker of Dutch. Note that no fricatives other than /f/ or /s / appeared in the stimuli. The fricative identification was part of a larger phoneme monitoring experiment with various phonemes as targets. Only the results for the fricative targets will be reported here.

We created 12 pseudowords with the target /f/ and 12 pseudowords with the target /s/. The fricatives were preceded and followed by / $\mathrm{a} \mathrm{i} \mathrm{u} /$. The target appeared always in the last syllable; stress was always on the first syllable. In addition, for every target fricative 12 filler items were created with the fricative in the penultimate syllable, and 12 filler items without the fricative.

The stimuli were recorded in a sound-attenuated room directly to computer and down-sampled to $22.05 \mathrm{kHz}$ (16 bit resolution). With Praat software cross-spliced and identityspliced versions of the pseudowords were created. Identityspliced fricatives were replaced by the same fricative taken from another token of the same pseudoword (e.g., /s/ in tikusa by /s / of another tikusa). Cross-spliced fricatives were replaced by the other fricative produced in the same context (e.g., /s/ in tikusa by /f/ from tikufa). Segmentation points for the fricatives were defined visually, on the basis of oscillograms and sonagrams. The end of harmonic structure of the preceding vowel and the beginning of harmonic structure in the fading noise of the fricative were defined as the splicing points. At zero-crossing points the coherent stochastic noise parts of the fricative were excised. The spliced stimuli were examined auditorily to ensure that no audible discontinuities had resulted from the manipulation.

\section{Procedure}

Participants sat in a sound-attenuated room in front of a computer screen, and heard both cross-spliced and identityspliced stimuli over headphones. Each pseudoword appeared only once in a session. Trials were blocked by target phoneme, with the order of blocks counterbalanced across participants. Participants were informed orally about the possible targets in advance; during the experiment a letter on the computer screen designated the current target. Participants were instructed to press a key immediately upon detecting in the nonword the sound represented by the displayed letter. Every target block of stimuli was followed by a break, the duration of which was controlled by the participants. From item onset, listeners had $2000 \mathrm{~ms}$ to respond. Failures to respond, and responses over $2000 \mathrm{~ms}$, were defined as timeout 
TABLE II. Average percentages of timeouts and mean RTs in milliseconds (ms) for the three languages and the two fricatives in both splicing conditions in experiment I. The absolute numbers of timeouts and the total numbers of trials are given in parentheses.

\begin{tabular}{cllll}
\hline \hline & \multicolumn{1}{c}{ Fricative } & Dutch & German & Spanish \\
\hline \multirow{2}{*}{ Mean } & /s/ identity spliced & $4.3 \%(4 / 93)$ & $1.8 \%(2 / 115)$ & $2.7 \%(4 / 170)$ \\
percentage & /s/ cross spliced & $4.3 \%(4 / 93)$ & $3.5 \%(4 / 115)$ & $2.7 \%(3 / 167)$ \\
of & /f/ identity spliced & $2.0 \%(2 / 93)$ & $1.8 \%(2 / 115)$ & $4.6 \%(6 / 169)$ \\
timeouts & /f/ cross spliced & $2.1 \%(2 / 93)$ & $1.0 \%(1 / 115)$ & $45.2 \%(55 / 145)$ \\
Mean RT & /s/ identity spliced & 488.22 & 440.82 & 544.04 \\
& /s/ cross spliced & 512.23 & 428.8 & 562.52 \\
& /f/ identity spliced & 531.27 & 442.22 & 618.6 \\
& /f/ cross spliced & 540.50 & 475.67 & 666.8 \\
\hline \hline
\end{tabular}

errors. The experiment was self-paced: The next stimulus was presented $1000 \mathrm{~ms}$ after the participant's response or timeout, and it was preceded by a beep tone.

\section{Participants}

Eighteen Dutch regular students, and 21 German and 23 Spanish exchange students from the Radboud University Nijmegen took part in this experiment. They were paid for their participation. None reported any speech or hearing disorders.

\section{B. Results}

Two items, one for each fricative target, were missed by more than $40 \%$ of the participants and therefore excluded from the analysis. The average timeouts (mean percentages of targets not correctly detected within $2000 \mathrm{~ms}$ ) and reaction times (RTs) for the remaining items for the three languages, the two fricatives and the two splicing conditions are shown in Table II.

\section{Timeouts}

We analyzed the timeouts by means of a loglinear analysis with the number of timeouts and nontimeouts for each stimulus as the dependent variable and language (Dutch, German, and Spanish), splicing (identity splicing and cross splicing), and fricative (/s/ and /f/) as independent variables. All main effects were significant (language: $F(2,129)$ $=30.22, p<0.001$; splicing: $F(1,127)=33.47, p<0.001$; and fricative: $F(1,128)=29.16, p<0.001)$. These main effects were modulated by an interaction between language and fricative $[F(2,125)=15.48, p<0.001]$. Importantly, we also observed the predicted interactions between language and splicing $[F(2,123)=6.63, p<0.001]$, and between language, fricative, and splicing $[F(2,120)=4.29, p<0.015]$. Splicing did not affect the number of timeout errors for the Dutch and German listeners, but the Spanish listeners were severely disturbed by misleading formant transitions $[F(1,41)$ $=48.42, p<0.001]$. The effect of splicing for Spanish was restricted to /f/ [interaction between splicing and fricative for Spanish $F(1,40)=11.32, p<0.001]$.

\section{RTs}

Latencies were measured from onset of the target fricative, defined as onset of the disharmonic structure in the stimulus wave form. Latencies below $150 \mathrm{~ms}$ were excluded from analysis $(0.3 \%$ of the data). Analyses of variance were conducted for participants $(F 1)$ and items $(F 2)$, with language, splicing, and fricative as independent variables.

The main effects of language and fricative were significant in both analyses [language: $F 1(2,58)=7.14, p<0.01$, $F 2(2,105)=55.42, p<0.001$; fricative: $F 1(1,174)=31.49, p$ $<0.001, F 2(1,21)=8.53, p<0.001]$, while splicing was significant only in the analysis by participants $[F 1(1,174)$ $=5.29, p<0.05]$. The interaction of language with fricative was significant in the analysis by participants $[F 1(2,174)$ $=31.60, p<0.001]$. More importantly, in the analysis by participants we also observed the interaction between language and splicing $[F 1(2,174)=5.12, p<0.01]$. This interaction failed to reach significance in the analysis by items.

\section{Summary and discussion}

We found language-specific patterns in the use of formant transitions in fricative identification. Only Spanish listeners were affected by misleading formant transitions. Apparently, they were attending to cues that were neglected by the Dutch and German listeners. Recall that the German and Dutch phoneme repertoires do not contain spectrally similar fricatives, while Spanish includes the two spectrally similar fricatives $/ \mathrm{f} /$ and $/ \theta /$. Even though $/ \theta /$ was not in the stimulus set, Spanish listeners paid attention to the formant transitions for /f/. They did not do so for /s/, which is spectrally distinct from the other fricatives in Spanish. These data support the hypothesis that listeners make use of formant transitions especially for fricatives that are spectrally similar to other fricatives in their native phoneme repertoire. Further, the results indicate that listeners do not necessarily take advantage of all acoustic information transmitted in the signal. The German and Dutch listeners showed no effects of the mismatching information that led Spanish listeners into errors.

However, Dutch participants had the advantage of listening to native phoneme realizations, while the Spanish listened to a foreign realization. The fact that German listeners showed the same pattern of results as the Dutch listeners may reflect a closer resemblance of German phonemes to Dutch than to Spanish phonemes. An alternative explanation for the cross-language differences might therefore be that listeners pay attention to more or to different cues when listening to a foreign pronunciation.

Wagner et al.: Language-specific cues for fricative identification 
TABLE III. Average percentages of timeouts and mean RTs in ms for the three languages and the two fricatives in both splicing conditions in experiment II. The absolute numbers of timeouts and the total numbers of trials are given in parentheses.

\begin{tabular}{cllll}
\hline \hline & \multicolumn{1}{c}{ Fricative } & Dutch & German & Spanish \\
\hline \multirow{2}{*}{ Mean } & /s/ identity spliced & $0 \%(0 / 180)$ & $2.2 \%(5 / 180)$ & $1.1 \%(2 / 172)$ \\
percentage & /s/ cross spliced & $0 \%(0 / 180)$ & $2.7 \%(4 / 180)$ & $0 \%(0 / 173)$ \\
of & /f/ identity spliced & $1.1 \%(2 / 180)$ & $1.1 \%(0 / 178)$ & $2.3 \%(4 / 172)$ \\
timeouts & /f/ cross spliced & $1.6 \%(3 / 180)$ & $2.2 \%(4 / 180)$ & $27.4 \%(47 / 173)$ \\
Mean RT & /s/ identity spliced & 461.54 & 474.34 & 474.93 \\
& /s/ cross spliced & 463.05 & 490.81 & 473.89 \\
& /f/ identity spliced & 550.67 & 569.06 & 601.93 \\
& /f/ cross spliced & 552.43 & 568.67 & 661.33 \\
\hline \hline
\end{tabular}

Experiment II was designed to test this second explanation. Experiments II and I differed principally in the native language of the speaker who recorded the stimuli: Dutch in experiment I, Spanish in experiment II. In experiment II, the Spanish listeners were thus presented with a familiar pronunciation, while the Dutch and German listeners were confronted with an unfamiliar realization of phonemes.

\section{EXPERIMENT II}

\section{A. Method}

\section{Materials and procedure}

The stimulus set from experiment I was now recorded by a native speaker of Spanish. In addition, 30 new fillers were created for each target with the target in the penultimate syllable or with the target missing. These fillers did not contain the phonemes /b/ and /d/, since Spanish phonotactics allows voiced bilabial and alveolar stops only in certain positions, and these consonants would therefore lead to a marked pronunciation by the Spanish speaker. The procedure was as in experiment $\mathrm{I}$.

\section{Participants}

Twenty-four Dutch regular, and 24 German and 24 Spanish exchange students from the Radboud University Nijmegen were paid to take part in this experiment. None had participated in experiment I, and none had any known speech or hearing disorders.

\section{B. Results}

We defined and analyzed timeout errors and reaction latencies in the same way as in experiment I. No data point was below $150 \mathrm{~ms}$, the common phoneme monitoring cutoff value (see, e.g., McQueen et al., 1999), and therefore no reaction time data were excluded from the analysis. Table III shows the results of this experiment.

\section{Timeouts}

All main effects were significant [language: $F(2,177)$ $=28.32, p<0.001$; splicing: $F(1,176)=28.49, p<0.001$; fricative: $F(1,175)=42.50, p<0.001]$. These main effects were modulated by interactions of language and splicing $[F(2,173)=5.39, p<0.001]$, language and fricative $[F(2,171)=13.68, p<0.001]$, and splicing and fricative
$[F(1,170)=6.3, p<0.005]$. The interaction between language, splicing, and fricative narrowly missed significance $[F(2,168)=2.4, p<0.1]$. Splicing affected the number of timeout errors for the Spanish listeners $[F(1,58)=38.4, p$ $<0.001$ ] only, and especially for the detection of /f/ [interaction of splicing and fricative for Spanish $F(1,56)$ $=10.41, p<0.001]$. These results replicate those of experiment I.

\section{RTS}

The main effects of language, splicing, and fricative were significant in both the participant and the item analyses [language: $F 1(2,58)=7.2, p<0.01, F 2(2,112)=11.56, p$ $<0.001$; splicing: $F 1(1,207)=5.79, \quad p<0.05, \quad F 2(1,140)$ $=4.94, \quad p<0.05 ;$ fricative: $F 1(1,207)=42.45, p<0.001$, $F 2(1,28)=25.45, p<0.001]$. Also the interaction of language and fricative was significant in both analyses $[F 1(2,207)=9.27, p<0.001, F 2(2,140)=8.63, p<0.001]$.

\section{Summary and discussion}

Experiment II further supports the hypothesis that Spanish listeners are affected by misleading formant transitions for fricative identification, while German and Dutch listeners are not. We ascribe these language differences to the different structures in the phoneme inventories of these languages, more precisely to the presence or absence of spectrally similar fricatives. Moreover, the finding that the Spanish only appeared to attend to formant transitions surrounding the labiodental fricative /f/ supports the hypothesis that the use of these cues is restricted to spectrally similar fricatives.

We obtained the same results for stimuli produced by a Dutch speaker (experiment I) and by a Spanish speaker (experiment II). Thus, experiments I and II together suggest that the native language of the speaker, or, in other words, the listeners' familiarity with the presented realization of the phonemes, does not alter the role of formant transitions in listeners' identification. We conclude that listeners also apply the native strategy when listening to a foreign pronunciation.

To explore further whether the presence of acoustically similar fricatives in a language's phoneme repertoire results in attention to formant transitions, we performed a third experiment with English native listeners. Since English is a Germanic language, it is in many respects more like Dutch and German than like Spanish. However, English has, like 
TABLE IV. Average percentages of timeouts and mean RTs in ms for the English listeners and the two fricatives in both splicing conditions in experiment III. The absolute numbers of timeouts and the total numbers of trials are given in parentheses.

\begin{tabular}{lcccc}
\hline \hline Fricative & $\begin{array}{c}\text { /s/ identity } \\
\text { spliced }\end{array}$ & $\begin{array}{c}\text { /s/ cross } \\
\text { spliced }\end{array}$ & $\begin{array}{c}\text { /f/ identity } \\
\text { spliced }\end{array}$ & $\begin{array}{c}\text { /f/ cross } \\
\text { spliced }\end{array}$ \\
\hline $\begin{array}{c}\text { Mean } \\
\text { percentage } \\
\text { of timeouts } \\
\text { Mean RT }\end{array}$ & $6.2(11 / 177)$ & $9.3(16 / 176)$ & $9.3(16 / 175)$ & $17.4(30 / 173)$ \\
\hline \hline
\end{tabular}

Spanish, both labiodental /f/ and the spectrally similar dental fricative $/ \theta /$ in its phoneme inventory. If our hypothesis is correct, English listeners should also attend to transitional cues, in particular for $/ \mathrm{f} /$.

\section{EXPERIMENT III}

\section{A. Method}

\section{Materials and procedure}

The materials were as in experiment II, i.e., the stimuli recorded by a native speaker of Spanish. The procedure and data analysis were as in the preceding experiments, with the exception that the target phoneme was not presented on screen. Grapheme-phoneme correspondences are often ambiguous in English; thus / f/ can be spelled as in "foal" or as in "phone," / s / can also be represented by the letter "c," as in "cedar," and the letter "s" can stand for /s/, as in "basic," for /z/, as in "cousin," or for nothing as in "debris." Therefore, we specified the target in recorded instructions at the beginning of every block of pseudowords, instead of in visual target representations.

\section{Participants}

Twenty-seven students from the participant pool of the Laboratory of Experimental Psychology of the University of Sussex took part in this experiment. They were native speakers of English and none reported any speech or hearing disorders.

\section{B. Results}

Mean timeouts and RTs are shown in Table IV.

\section{Timeouts}

Both splicing (cross-spliced versus identity-spliced items) and fricative (/s/ versus /f/) were significant [splicing: $F(1,58)=5.76, p<0.05$; fricative: $F(1,57)=5.95, p$ $<0.05]$. The interaction did not reach significance. The English listeners missed more items in the cross-spliced condition, and more /f/ than /s/.

\section{RTs}

$0.4 \%$ of the data was below $150 \mathrm{~ms}$, and was excluded from the analysis. Only fricative was significant in both analyses $\quad[F 1(1,78)=12.66, p<0.001, F 2(1,56)=2.89, p$ $<0.05]$. Listeners responded less rapidly to $/ \mathrm{f} /$ than to $/ \mathrm{s} /$.

\section{Summary and discussion}

English listeners also appear to pay attention to formant transitions. The crucial interaction between fricative and splicing was not significant, and therefore at this point we cannot decide with certainty whether English listeners make use of transition cues only for identification of /f/. However, the data suggest that English listeners, like Spanish listeners, are particularly affected in the case of / f/ (note that the effect of cross splicing, though statistically robust for both fricatives for these listeners, was twice as strong in the timeout errors for /f/ as for /s / $-87 \%$ increase as opposed to $47 \%$ ). Both English and Spanish listeners have learnt to distinguish between /f/ and / $\theta /$, two highly confusable fricatives. This apparently made them more attentive to the additional acoustic cues in the formant transitions.

Previous research has shown that the labiodental fricative is hard to identify on the basis of spectral characteristics alone (Harris, 1958; Jongman et al., 1998). So far we have shown that some listeners attend to transitional cues for this fricative. Our hypothesis, however, is that listener's use of transitional information in fricative identification reflects not just inherent distinctiveness of fricatives, but the presence of spectrally confusable pairs in the native fricative inventory. On this hypothesis, even fricatives which are generally easy to identify should encourage use of transitional information in a language which contains more fricatives with similar spectra.

The /s/ has been shown to be perceptually very salient because of the acoustic make-up of its noise spectrum (Wang and Bilger, 1973). During the articulation of /s/ air jets are created as the airflow passes the edges of the teeth; this results in relatively high intensity peaks in the high-frequency range of the spectrum, which serve as reliable cues and make this fricative acoustically robust. Listeners should nevertheless also exploit formant transitions to identify /s/, we predict, if other fricatives are close to /s/ in their native perceptual space.

We tested this in Polish, which has 11 fricatives

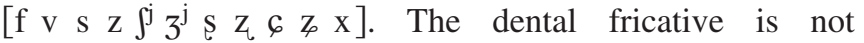
present, so that $/ \mathrm{f} /$ is acoustically distinct from all other fricatives. The presence of the postalveolar, alveolopalatal, and palatal retroflex fricatives may, however, reduce the perceptual saliency of /s/. In acoustic terms, the /s/ typically has energy peaks in the frequency range between 3 and $7 \mathrm{kHz}$. The postalveolar $/ \int /$ exhibits energy peaks in the frequencies between 1.5 and $5 \mathrm{kHz}$, while the Polish alveolopalatal / 6 / has its energy maxima in the range between 2 and $6 \mathrm{kHz}$. Finally, the retroflex Polish fricative shows its high energy peaks around 1 and $4 \mathrm{kHz}$ (Jassem, 1968). This concentration of several fricatives with energy distributions in the same spectral range might hinder the identification of these fricatives in Polish. We therefore expect Polish listeners to pay attention to formant transitions for $/ \mathrm{s} /$.

Wagner et al:: Language-specific cues for fricative identification 
TABLE V. Average percentages of timeouts and mean RTs in ms for the Polish listeners and the two fricatives in both splicing conditions in experiment IV. The absolute numbers of timeouts and the total numbers of trials are given in parentheses.

\begin{tabular}{ccccc}
\hline \hline Fricative & $\begin{array}{c}\text { /s/ identity } \\
\text { spliced }\end{array}$ & $\begin{array}{c}\text { /s / cross } \\
\text { spliced }\end{array}$ & $\begin{array}{c}\text { /f/ identity } \\
\text { spliced }\end{array}$ & $\begin{array}{c}\text { /f/ cross } \\
\text { spliced }\end{array}$ \\
\hline $\begin{array}{c}\text { Mean } \\
\text { percentage of } \\
\text { timeouts } \\
\text { Mean RT }\end{array}$ & $5.5(10 / 180)$ & $12.7(23 / 180)$ & $0(0 / 180)$ & $3.3(6 / 180)$ \\
\hline \hline
\end{tabular}

\section{EXPERIMENT IV}

\section{A. Method}

\section{Materials and procedure}

Materials were as in experiments II and III, procedure was as in experiment II, and data analysis was as in all the preceding experiments.

\section{Participants}

Twenty-four students at the Uniwersytet Śląski in Katowice, all native Polish speakers, were paid to take part in this experiment. None reported any speech or hearing disorders.

\section{B. Results}

Table V shows the average timeouts and RTs.

\section{Timeouts}

Both main effects were again significant: splicing $[F(1,58)=10.19, p<0.01]$ and fricative $[F(1,57)=21.92, p$ $<0.001]$. The interaction between fricative and splicing narrowly failed to reach significance $[F(1,56)=3.73, p<0.06]$. More timeouts occurred for the cross-spliced items, and for /s/ $(9.16 \%$ versus $1.6 \%$ for $/ \mathrm{f} /)$. Furthermore, the effect of splicing appeared smaller for /f/ than for /s/.

\section{RTs}

The main effect of fricative was significant in the analysis by participants only $[F 1(1,69)=5.65, p<0.05]$. As Table $\mathrm{V}$ shows, the Polish RTs were relatively long.

\section{Summary and discussion}

Like Spanish and English listeners, Polish listeners are affected by misleading formant transitions. The phoneme repertoires of all three languages contain spectrally similar fricatives, and the results are thus in line with our hypothesis that listeners learn to direct their attention to subtle acoustic cues for fricative identification if required by their native phoneme repertoire. Furthermore, we can reject the possibility that listeners only take advantage of formant transitions in order to identify the spectrally diffuse and therefore perceptually less salient labiodental fricative. Even though we found no significant interaction between splicing and fricative for Polish listeners, the error data indicate that in contrast to all the other listener groups Polish listeners missed four times as many cross-spliced /s/ items than /f/ items.
Especially the spectrally salient /s/ requires attention to formant transitions if this fricative can easily be confused with other fricatives in the listeners' phoneme repertoire.

On which level may such language-specific differences occur? We used the term attention to refer to listeners' learned selection of acoustic cues for phoneme identification, without assuming that listeners differ in sensitivity at the auditory level. Differences in sensitivity would imply that Dutch and German have "lost" such sensitivity. However, listeners are known to display sensitivity to foreign-language contrasts which fall entirely outside the range of the native phoneme repertoire (Best, McRoberts, and Sithole, 1988). Thus the effects that we have observed may reflect strategic listening choices which have no implications for the underlying sensitivity. If so, Dutch listeners, too, may perceive the acoustic mismatches if their attention is drawn to them. We tested this possibility in experiment $\mathrm{V}$.

Furthermore, the phoneme inventories we have tested differ in whether or not they offer an alternative category in the case of an ambiguous fricative of a particular kind. In experiment $\mathrm{V}$ we also tested the effects of this response availability. We used an untimed open-choice identification task, with Dutch and Spanish listeners. If no response alternatives are given, participants are expected to choose a phoneme category from their native inventory. Spanish listeners may identify at least some of the cross-spliced /f/ tokens as $|\theta|$. Dutch listeners, in contrast, should identify all tokens of cross-spliced /f/ as /f/. By asking subjects to judge the goodness-of-fit of the stimuli, we examined the extent to which both Dutch and Spanish listeners perceive mismatch effects of cross splicing.

\section{EXPERIMENT V}

\section{A. Method}

\section{Materials}

Materials were the target-bearing VCV-strings of all 60 items used in experiment II, including the identity-spliced and cross-spliced targets (e.g., from the experimental item tikufa we presented the fragment $u f a$ ).

\section{Procedure}

Participants, seated in a sound-attenuated room, were presented with the VCVs over headphones. They were instructed to write down the intervocalic consonant, and to judge on a scale from 1 to 8 whether it was a poor or a good example of this consonant. After the test, participants identified the letters they used to describe the consonants by writing down a native example word containing each letter used.

\section{Participants}

Thirty-one students from the Radboud University Nijmegen took part in this experiment. Fourteen were native Dutch regular students, and 17 were native Spanish exchange students. They were paid for their participation. None reported any speech or hearing disorders. 


\section{B. Results}

Dutch listeners always identified each of the stimuli as either /f/ or /s/. Spanish listeners, on the other hand, showed greater response variance. Five of the 17 Spanish listeners reported hearing exclusively /f/ and /s/, while the remaining 12 participants included other consonants in their responses. All cross-spliced /s / were identified as /s/, but the responses for /f/ varied, including $/ \mathrm{b} /, / \mathrm{d} /, / \mathrm{m} /$ and, most frequently, the dental fricative $/ \theta /$. One item was identified by none of these 12 Spanish participant as /f/, but as a poor example of $\mid \theta /$. All in all nine cross-spliced /f/ were identified by at least five Spanish participants as a consonant belonging to a category other than /f/.

The average ratings for the items which were correctly identified as either an /s / or an /f/ were: for identity-spliced /s/, Dutch 3.95, Spanish 4.81; for cross-spliced /s/, Dutch 3.94, Spanish 4.67; for identity-spliced /f/, Dutch 3.78, Spanish. 4.53; for cross-spliced /f/, Dutch 3.01, Spanish 3.73. We analyzed the averaged ratings in an analysis of variance. We found main effects of language $[F(1,56)$ $=120.77, p<0.001]$, splicing $[F(1,56)=21.96, p<0.001]$, and fricative $[F(1,56)=37.01, p<0.001]$ and an interaction between splicing and fricative $[F(1,56)=15.25, p<0.001]$. In general Spanish listeners rated the stimuli as better examples than Dutch listeners, probably because they were presented with their native phoneme realizations. The crossspliced /f/ items were rated as poorer examples than the identity-spliced /f/ by both listener groups.

\section{Discussion}

Experiment $\mathrm{V}$ showed that the acoustic mismatch in the cross-spliced /f/ tokens turned them into poorer instances of /f/. While Dutch listeners just perceived these /f/ tokens as poorer members of the /f/ category, Spanish listeners identified some of these tokens as belonging to another category, most frequently as a $/ \theta /$. Thus the availability of an alternative category may be a crucial factor in determining whether the mismatch between fricative noise and formant transitions results in the perception of a different category. Although Dutch listeners seem to accept the cross splicing as allophonic variation of $/ \mathrm{f} /$, the goodness ratings showed that they too were sensitive to the acoustic mismatch.

We reanalyzed the timeout errors from experiment II, including for /f/ only the six items which the Spanish participants had always identified as /f/ when cross spliced. In this new analysis, the significant three-way interaction between language, splicing, and fricative no longer reached significance. This may be because that three-way interaction had been principally carried by the nine items which produced variable responses in experiment V; alternatively, of course, it could simply result from reduction of statistical power.

In an additional analysis we included the average Dutch ratings as a predictor for the Spanish timeout errors in experiment II. Splicing remained statistically significant $[F(1,57)=42.12, p<0.001]$. This result suggests that even though Dutch listeners perceive the acoustic manipulation in the stimuli, the cross splicing of the /f/ is definitely more harmful for the Spanish than for the Dutch listeners.

\section{GENERAL DISCUSSION}

Many studies have investigated the contribution of formant transitions to fricative identification. Some studies reported robust effects whereas others failed to find any perceptual relevance of formant transitions for fricatives. In four phoneme detection experiments, we tested the hypothesis that attention to formant transitions as cues for fricative identification differs as a function of the presence of perceptually confusable fricatives in the listeners' native language. The targets in the detection experiments were /s/ and /f/ surrounded by either misleading (cross-splicing condition) or by coherent (identity-splicing condition) formant transitions. The stimuli were presented to Dutch, German, Spanish, English, and Polish listeners.

Our results support the hypothesis. First, target fricatives surrounded by misleading formant transitions were missed more often than fricatives with coherent formant transitions. This finding confirms previous work (Harris, 1958; Heinz and Stevens, 1961) showing that English listeners attend to formant transitions for some fricatives. More importantly, however, we observed a language-specific pattern of taking these acoustic cues into account for phoneme identification. Native listeners of Dutch and German, both languages without spectrally confusable fricatives, were not affected by misleading formant transitions. In contrast, listeners of Spanish and English, languages with the spectrally similar labiodental /f/ and dental / $\theta /$ fricatives, and Polish, a language with spectrally similar sibilants, were affected by misleading formant transitions.

On the basis of the languages in which we found formant transitions to be used, we further queried whether attention to formant transitions is restricted to the spectrally similar contrasts only or whether it generalizes to nonconfusable fricatives. We found that transition cues were restricted to /f/ for the Spanish listeners. For Polish listeners, the crucial interaction between splicing and fricative narrowly failed to reach significance $(p=0.053)$. But, as shown in Table V, the effect of splicing was greater for /s/ than for /f/. For English, the interaction between splicing and fricative did not reach significance, even though the effect is numerically greater for /f/ than for /s/. This may indicate that English listeners were also affected by misleading formant transitions for /s/. This is not incompatible with our hypothesis, if we take into consideration that English, in contrast to Spanish, has a postalveolar fricative category, which is spectrally more similar to /s/ than to /f/. Thus, with respect to our second hypothesis, we can tentatively conclude that attention to formant transition is restricted to spectrally similar fricative categories. Which fricatives are spectrally similar, of course, is a function of all fricative contrasts in a language, and their distribution in the perceptual space.

The pattern in our data, and in English in particular, might of course also have been affected by the particular splicing manipulation we applied to our stimuli. The frication noises of /f/ and /s / differ in several ways; most impor- 
tantly, /f / has a flat diffuse spectrum, while /s / shows prominent energy peaks. The spectra of /f/ and $/ \theta /$, and of /s/ and $/ \mathrm{S} /$, however, show more similarities; cross splicing within these pairs might well show effects with English listeners. Whalen (1981) found that English listeners' categorization of an ambiguous synthetic fricative noise as either /s/ or $/ \mathrm{S} /$ was influenced by formant transitions. In his experiment, a synthetic ten-step noise continuum was combined with coherent or inappropriate natural vocalic portions, including formant transitions. Interestingly, the formant transitions contributed to listeners' decision only at those steps of the noise continuum which modeled noise spectra with energy peaks appropriate for natural $/ \mathrm{J} /$ or $/ \mathrm{s} / \mathrm{spectra}$. This suggests that for English listeners cross spliced stimuli containing fricative noise with more defined spectral peaks (as $/ \mathrm{S} /$ ) in combination with mismatching formant transitions may lead to a similar effect for /s/, as found mainly for /f/. In our study, however, the difference between the cross-spliced pairs apparently overrode a potential confusion for the English listeners. Furtherresearch could investigate whether mismatching information in formant transitions to /s/ might also mislead English listeners-for example, into classifying an input as post-alveolar.

Importantly, the Polish data suggest that the acoustic make-up of a fricative by itself does not determine the use of formant transitions. Even though /s/ has salient acoustic characteristics (Harris, 1958; Strevens, 1960; Jassem, 1965) which make it perceptually very robust, Polish listeners were affected in particular for this fricative. Thus, the crucial factor in the use of formant transitions appears to be the acoustic make-up of a fricative in relation to all other fricatives in the phoneme inventory.

The present results indicate that listeners integrate cues in a language-specific way. The information conveyed in formant transitions appears to play a crucial role in determining fricative categorization for Spanish, English, and Polish listeners. This language-specific way of selecting cues for attention does not seem to be a strategy that a listener can easily adapt to the requirements of the situation, or to the experimental situation. The stimulus set in our experiments did not contain the dental fricative $|\theta|$. That is, a direct distinction between the two confusable fricatives $/ \mathrm{f} /$ and $/ \theta /$ was not necessary for efficient performance within the experimental situation. Nonetheless, the Spanish and English listeners were substantially misled by incorrect formant transitions for /f/. Similarly, the Polish listeners were misled by incorrect formant transitions for $/ \mathrm{s} /$, even though the palatal fricatives, which in Polish might be confused with /s/, were not present in the experiment. This suggests that for listeners of these languages, formant transitions are part and parcel of the fricative categories.

We have distinguished "attention" from "sensitivity" to formant transitions. Experiment V showed that Dutch listeners perceive an acoustic difference between the identity- and cross-spliced items. They rated cross-spliced /f/ tokens as poorer examples of /f/, though in phoneme monitoring these poorer examples were not responded to significantly differently from the better examples. We assume that the attunement to a native language does not have any consequences on a low auditory level: sensitivity is unaffected. All listeners may perceive acoustic mismatches between formant transitions and noise spectrum, but language experience determines whether this information is attended to in fricative identification. Experiment V shows that the mismatching information in the transitions led Spanish listeners into the percept of a different fricative; the availability of more fricative categories encourages attention to subtle cues such as formant transitions. Where there is no alternative category-as in the case of Dutch-mismatching information in formant transitions may be treated as just allophonic variation. Thus what Spanish listeners in experiment V could identify as a dental fricative or even as a stop, Dutch listeners simply judged to be $/ \mathrm{f} /$. The number of possible choices for identifying an ambiguous stimulus has an effect on the distinctiveness of categories, and thus on listeners' response options. Recall that the goodness ratings of the Dutch listeners in experiment $\mathrm{V}$ did not suffice to explain the errors made by Spanish listeners, however. Thus the Dutch and Spanish listeners differed in how mismatching information affected fricative identification.

Primary cues are defined by some researchers (e.g., Stevens and Blumstein, 1981) as invariant acoustic properties which are independent of the phonetic context and sufficient to evoke the percept of a given phoneme. Secondary cues, in contrast, are context-dependent cues, exploited by listeners to support primary cues when needed, for instance in difficult listening conditions. We have shown that a context-dependent cue can also make an important and systematic contribution to fricative identification. Spanish listeners missed over $25 \%$ of the /f/ tokens which were surrounded by misleading formant transitions. The selection of primary and secondary cues appears to be language and phoneme specific, and depends on the degree to which cues enable listeners to distinguish native phoneme categories accurately and efficiently. Even though other acoustic characteristics, such as the generally higher intensity of the fricative noise, are used by listeners to distinguish sibilants from other fricatives, Polish listeners appear to use cues in the formant transitions, simply because of the number of confusable sibilants in their native phoneme repertoire.

In our experiments, listeners did not categorize or discriminate pairs of fricatives. In phoneme monitoring, participants react as soon as they recognize the target, and they do so only if the acoustic stimulus matches their abstract memory of the target. Reduced or mismatching information-here, the cross-spliced formant transitionsled Spanish, English, and Polish listeners into errors. Most previous studies of fricative perception have used untimed identification tasks. Results showed that Argentinian listeners could use transition information for some fricative contrasts (Borzone de Manrique and Massone, 1981), Dutch listeners apparently did not use it (Klaassen-Don, 1983), while English listeners appeared to use transition information in some studies (Harris, 1958) but not in others (Jongman, 1989). We cannot exclude the possibility that with unlimited response time listeners may be able to extract more information from static cues than they do in a running-speech situation, and that characteristics of particular experiments may have been 
more versus less encouraging to such strategies. A task such as categorization (Whalen, 1981) ${ }^{1}$, for example, could induce a different listening strategy; in categorization, listeners assign an acoustic signal to one or another category, and it is reasonable to assume that the mental representations of these categories, including the acoustic cues which distinguish between them, are in listeners' focus of attention, and might not need to be retrieved with every stimulus. This could affect both response accuracy and reaction times.

Adult listeners are specialized in identifying their native phonemes. An efficient way of selecting acoustic cues is thus another feature of language-specific processing which children must acquire in the course of their language development. In the same way that children learn to distinguish only native language contrasts (e.g., Werker and Tees, 1999; Sebastián-Gallés and Soto-Faraco, 1999), children must learn to be parsimonious with their attention to the subtle details of the acoustic signal and with the selection of relevant cues. Research by Nittrouer and colleagues (Nittrouer and Miller, 1997a, 1997b; Nittrouer, 2002) shows that there is indeed a developmental shift in the relevance of the cues conveyed by the frication and by the dynamics in the formant transitions for fricative identification. American English speaking children between 4 and 7 years of age show a developmental decrease in their weighting of formant transitions and a developmental increase in their weighting of the noise characteristics for $/ \mathrm{s} /$ and $/ \mathrm{S} /$. On the other hand, another study by Nittrouer (2002) showed that American English speaking children and adults are more similar in assigning weight to formant transitions for the distinction between the labiodental and the dental fricatives. Thus, the developmental shift is restricted to the contrasts which are sufficiently characterized by the static cues alone. Nittrouer argues that the attention/sensitivity to dynamic cues diminishes when children learn which cues carry "phonetic informativeness" in their native language.

Children's speech perception differs even up to 10 years of age from adults' speech perception (Elliot and Katz, 1980). Nittrouer's developmental weighting shift theory contrasts with, for instance, explanation in terms of auditory cortex maturation (Sussman, 2001). Most of the data relevant to this debate come so far from English, and we suggest that the debate would profit from additional data from other languages, for instance, the five languages of the present study. Our results show that children will reorganize their sensitivity to formant transitions in a language specific way to spectrally similar fricatives. English, Spanish, and Polish children should keep their attention to formant transitions, whereas Dutch and German children will not.

The shift in attention during language development entails that a listener would have to reacquire, or reorganize attention to these cues in order to attain a native-like perception in a second language. Previous research (Repp, 1981; Hazan, Iverson, and Bannister, 2005) suggests that listeners can indeed direct attention to otherwise unused phonetic cues, at least after being exposed to sufficient training. Future research will have to determine how rapidly speakers of a language without perceptually similar fricatives can learn to take advantage of formant transitions to efficiently distinguish between perceptually similar fricatives in a second language.

Are fricatives perceived only on the basis of the static characteristics of their fricative spectrum, or do formant transitions also play a role? A large number of studies have addressed these questions, but the pattern of results, as we demonstrated in the Introduction, has been contradictory. Previous studies have examined the question in different languages; and language-specific phonology may be the key to whether listeners rely solely on spectral cues to fricative identity, or also attend to transition information. Even though all listeners will always make use of information in the fricative spectrum, for listeners of some languages formant transitions also play a crucial role for some of their native fricatives. Mismatching acoustic information in formant transitions may be perceived by all listeners at a low phonetic level, but the use of this information for the identification of a given fricative seems to depend on whether the spectral characteristics of its frication suffice to distinguish this fricative from all other fricatives in the listener's language.

\section{ACKNOWLEDGMENTS}

The authors are grateful to Professor Alan Garnham from the University of Sussex and to Dr. Jolanta Tambor from Uniwersytet Śląski in Katowice for their help in conducting the experiments in England and in Poland. They would further like to thank James McQueen for his helpful comments on an earlier version of this text. This research was supported by the NWO SPINOZA project "Native and Non-Native listening."

${ }^{1}$ Note that Whalen (1981) research also showed effects of context vowels on the identification of fricatives. We in fact included the context vowels as factors into our analyses. As these results did not prove to be language specific, however, we do not report them in detail.

Best, C. T., McRoberts, G. W., and Sithole, N. M. (1988). "Examination of perceptual reorganization for nonnative speech contrasts: Zulu click discrimination by English-speaking adults and infants," J. Exp. Psychol. Hum. Percept. Perform. 14, 45-60.

Borzone de Manrique, A. M., and Massone, M. I. (1981). "Acoustic analysis and perception of Spanish fricative consonants," J. Acoust. Soc. Am. 69(4), 1145-1153.

Connine, C., and Titone, D. (1996). "Phoneme monitoring," Lang. Cognit. Processes 11, 635-645.

Costa, A., Cutler, A., and Sebastián-Gallés, N. (1998). "Effects of phoneme repertoire on phoneme decision," Percept. Psychophys. 60, 1022-1031.

Elliot, L. L., and Katz, D. (1980). "Children's pure-tone detection," J. Acoust. Soc. Am. 67, 343-344.

Fruchter, D., and Sussman, H. (1997). "The perceptual relevance of locus equations," J. Acoust. Soc. Am. 102(5), 2997-3008.

Hamann, S. (2003). The Phonetics and Phonology of Retroflexes, (LOT, Utrecht).

Harris, K. S. (1958). "Cues for the discrimination of American English fricatives in spoken syllables," Lang Speech 1, 1-7.

Hazan, V., Iverson, P., and Bannister, K. (2005). "The effect of acoustic enhancement and variability on phonetic category learning by L2 learners," Proceedings of the ISCA Workshop on Plasticity in Speech Perception, London, UK, June 2005.

Heinz, J. M., and Stevens, K. N. (1961). "On the properties of fricative consonants," J. Acoust. Soc. Am. 33, 589-593.

Jassem, W. (1965). "Formants of fricative consonants," Lang Speech 8, $1-16$.

Jassem, W. (1968). "Acoustic description of voiceless fricatives in terms of 
spectral parameters," in Speech Analysis and Synthesis, edited by W. Jassem, Panstwowe Wydawnictwo Naukowe, Warsaw, pp. 189-206.

Jassem, W. (2003). "Illustrations of the IPA: Polish," J. Int. Phonetic Assoc. 33(1), 103-107.

Jongman, A. (1989). "Duration of fricative noise required for identification of English fricatives," J. Acoust. Soc. Am. 85, 1718-1725.

Jongman, A., Sereno, J., Wayland, R., and Wong, S. (1998). "Acoustic properties of English fricatives,” J. Acoust. Soc. Am. 103, 3086.

Jongman, A., Wayland, R., and Wong, S. (2000). "Acoustic characteristics of English fricatives," J. Acoust. Soc. Am. 108(3), 1252-1263.

Klaassen-Don, L. E. O. (1983). "The influence of vowels on the perception of consonants," Doctoral dissertation, Leiden University (unpublished).

LaRiviere, C., Winitz, H., and Herriman, E. (1975). "The distribution of perceptual cues in English prevocalic fricatives,” J. Speech Hear. Res. 18, 613-622.

Martin, J. G., and Bunnell, H. T. (1981). "Perception of anticipatory coarticulation effects," J. Acoust. Soc. Am. 69, 559-567.

McQueen, J. M., Norris, D., and Cutler, A. (1999). "Lexical influence in phonetic decision making: Evidence from subcategorical mismatches," J. Exp. Psychol. Hum. Percept. Perform. 25, 1363-1389.

Nittrouer, S. (2002). "Learning to perceive speech: How fricative perception changes, and how it stays the same," J. Acoust. Soc. Am. 112(2), 711719 .

Nittrouer, S., and Miller, M. E. (1997a). "Developmental weighting shifts for noise components of fricative-vowel syllables," J. Acoust. Soc. Am. 101(1), 572-580.

Nittrouer, S., and Miller, M. E. (1997b). "Predicting developmental shifts in perceptual weighting schemes," J. Acoust. Soc. Am. 101(4), 2253-2266.
Otake, T., Yoneyama, K., Cutler, A., and van der Lugt, A. (1996). "The representation of Japanese moraic nasals," J. Acoust. Soc. Am. 100(6), $3831-3842$.

Repp, B. (1981). "Two strategies in fricative discrimination," Percept. Psychophys. 30(3), 217-227.

Sebastián-Gallés, N., and Soto-Faraco, S. (1999). "Online processing of native and non-native phonemic contrasts in early bilinguals," Cognition 72, 111-123.

Stevens, K. N. (1998). Acoustic phonetics (MIT Press, Cambridge, MA).

Stevens, and Blumstein, S. E. (1981). "The search for invariant acoustic correlates of phonetic features," in Perspectives on the Study of Speech, edited by P. L. Miller (Erlbaum, Hillsdale, NJ) pp. 1-38.

Strevens, P. (1960). "Spectra of fricative noise in human speech," Lang Speech 3, 32-49.

Sussman, H. (2001). "Vowel perception by adults and children with normal language and specific language impairment: Based on steady states or transitions?," J. Acoust. Soc. Am. 109(3), 1173-1180.

Wang, M. D., and Bilger, R. C. (1973). "Consonant confusions in noise: A study of perceptual features," J. Acoust. Soc. Am. 54, 1248-1266.

Weber, A. (2001). "Help or hindrance: How violation of different assimilation rules affects spoken-language processing," Lang Speech 44, 95-118.

Werker, J. F., and Tees, R. C. (1999). "Influences on infant speech processing: toward a new synthesis," Annu. Rev. Psychol. 50, 509-535.

Whalen, D. H. (1981). "Effects of vocalic formant transitions and vowel quality on the English [s]-[̌̌] boundary," J. Acoust. Soc. Am. 69, 275282

Zygis, M., and Hamann, S. (2003). "Perceptual and acoustic cues of Polish coronal fricatives," Proceedings of the 15th ICPhS, pp. 395-398. 